\title{
REFERENCES
}

Anson, M. L. \& Mirsky, A. E. (1928). J.gen. Physiol.12, 273.

Davies, T. H. (1940). J. biol. Chem. 135, 597.

Dhéré, Ch. \& Vegezzi, G. (1916). C.R. Acad. Sci., Paris, 163, 209.

Drabkin, D. L. (1942). J. biol. Chem. 146, 605.

Fischer, H. \& Orth, H. (1934). Die Chemie des Pyrrols, 2. Leipzig: Akademische Verlagsgesellschaft.

Gouy, G. (1889). C.R. Acad. Sci., Paris, 109, 935.

Hartley, E. G. J. (1928). J. chem. Soc. p. 780.

Hill, R. (1926). Proc. Roy. Soc. B, 100, 419.

Hill, R. (1929). Proc. Roy. Soc. B, 105, 112.

Hogness, T. R., Zscheile, F. P., Sidwell, A. E. \& Barron, E. S. G. (1937). J. biol. Chem. 118, 1.
Keilin, J. (1943). Biochem. J. 37, 281.

Keilin, J. (1944). Nature, Lond., 154, 120.

Pauiing, L. \& Coryell, C. D. (1936). Proc. nat. Acad. Sci., Wash., 22, 159.

Robbie, W. A. \& Leinfelder, P. J. (1945). J. industr. Hyg. 37, 136.

Schalfejeff, M. (1885). Abstract in Ber. dtsch. chem. Ges. 18 (Referat Bd.), 232.

Toda, S. (1926). Biochem. Z. 172, 17.

Warburg, O., Negelein, E.\& Christian, W.(1929). Biochem.Z. 214, 26.

Wyman, J. (1948). Advanc. protein Chem. 4, 407.

Zeynek, R. von (1910). Hoppe-Seyl. Z. 70, 224.

\section{On the Properties and Nature of Dihydroxyl-haem}

\author{
By JOAN KEILIN, The Biochemical Laboratory, University of Cambridge
}

(Received 2 May 1949)

Dhéré \& Vegezzi (1916), while working on the reduction of haematin under different conditions, described a compound obtained by reducing an ethanolic solution of haematin in strong alkali with sodium dithionite $\left(\mathrm{Na}_{2} \mathrm{~S}_{2} \mathrm{O}_{4}\right)$ in air. This compound, which they named 'alkaline haemochromogen', was red in colour and showed a characteristic absorption spectrum consisting of two bands, of which the $\beta$-band lying nearer the blue end of the spectrum was much stronger than the $\alpha$-band. It was not until several years later, however, that the term 'haemochromogen' was defined by Anson \& Mirsky (1925) as covering the compounds of reduced haematin or haem with different nitrogenous substances, and Hill (1926) showed conclusively that in a haemochromogen $2 \mathrm{~mol}$. of an organic base are combined with the one iron atom of haem. Of all the nitrogenouis compounds examined only potassium cyanide and methyl isocyanide (methyl carbylamine) were found to form two distinct compounds with haem: monocyan-haem and dicyan-haem, which contain 1 and 2 mol. of cyanide per molecule of haem respectively, and mono- and di-carbylamine haem (see Keilin, 1949).

The object of this investigation is to elucidate the nature of the compound described by Dhéré \& Vegezzi as 'alkaline haemochromogen' and to compare it with haem, monocyan-haem, dicyanhaem and haemochromogens. It is proposed to describe the 'alkaline haemochromogen' of Dhéré \& Vegezzi under the name of dihydroxyl-haem, with a prefix to the haem denoting the type of porphyrin used; e.g. dihydroxyl-protohaem and dihydroxylurohaem.

\section{MATERIAL AND METHODS}

Protohaemin. This was prepared by the method of Schalfejeff (1885) from ox or horse blood.

Haematohaemin. Haematoporphyrin was prepared from protohaemin by Nencki's method (Nencki \& Seiber, 1888) and the iron was introduced by treating the porphyrin with $\mathrm{FeSO}_{4}$ in the presence of glacial acetic acid and sodium acetate.

Urohaemin. Uroporphyrin I was isolated from the urine of a case of congenital porphyria. The porphyrin was esterified and the urohaemin prepared from the octamethyl ester according to Fischer \& Orth (1934).

Spectroscopic observations. For all qualitative spectroscopic examinations and for preliminary quantitative experiments a microspectroscope was used as previously described (Keilin, 1943). The recording of absorption spectra in the visible and violet regions was carried out with a Beckman photoelectric spectrophotometer. In all figures the molecular absorption coefficients ( $\epsilon)$, as previously defined (Keilin, 1949), were plotted against wavelengths (in $\mathrm{m} \mu$.).

\section{RESULTS}

\section{The reaction of protohaem with sodium hydroxide}

A solution of protohaem, obtained by dissolving protohaemin in $0.1 \mathrm{~N}-\mathrm{NaOH}$ or $1 \%(\mathrm{w} / \mathrm{v}) \mathrm{Na}_{2} \mathrm{CO}_{3}$ (anhydrous) and reducing it with $\mathrm{Na}_{2} \mathrm{~S}_{2} \mathrm{O}_{4}$, shows two very diffuse absorption bands. The addition of ethanol to this solution affects this spectrum only in so far as it causes a very slight sharpening of the bands. If, however, the ethanolic solution of protohaematin contains a much higher concentration of $\mathrm{NaOH}$, the solution becomes cherry coloured on reduction, and a characteristic two-banded spectrum is seen in which the $\beta$-band, with its centre at 
$558 \mathrm{~m} \mu$., is much stronger than the $\alpha$-band which lies at $590 \mathrm{~m} \mu$. (Fig. 1). The $\gamma$-band of this compound

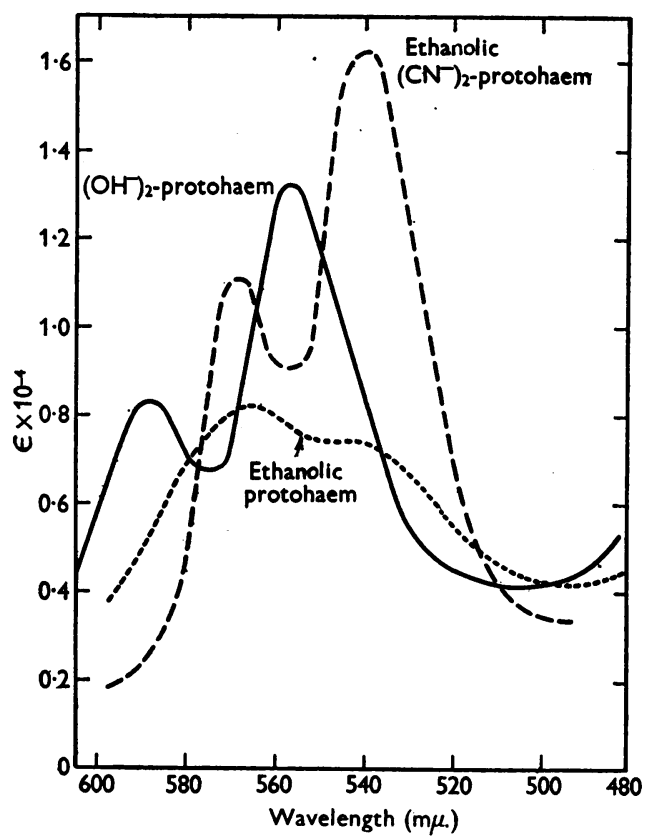

Fig. 1. Absorption bands in the visible region of the spectrum of $\left(\mathrm{OH}^{-}\right)_{2}$-protohaem, $\left(\mathrm{CN}^{-}\right)_{2}$-protohaem containing $50 \%$ $\mathrm{v} / \mathrm{v}$ ethanol (protohaem $=1.85 \times 10^{-5} \mathrm{M}$ ) and ethanolic protohaem. (Protohaem $=1.65 \times 10^{-4} \mathrm{M}, l=0.5 \mathrm{~cm}$.)

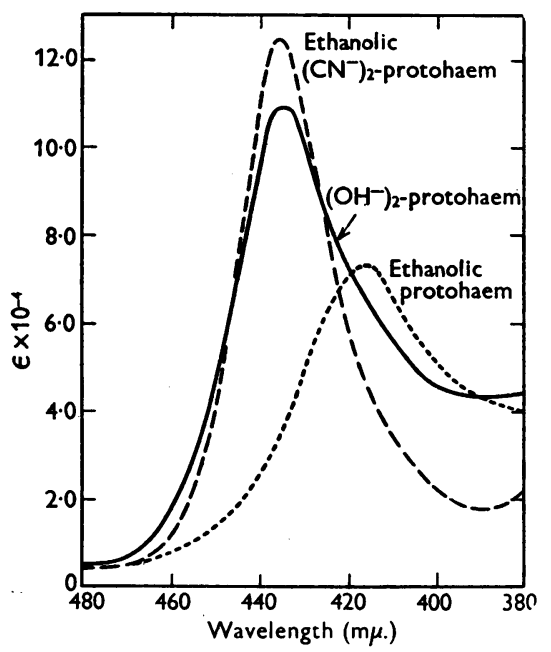

Fig. 2. Absorption spectra showing the $\gamma$-bands of $\left(\mathrm{OH}^{-}\right)_{2-}$ protohaem, ethanolic protohaem and $\left(\mathrm{CN}^{-}\right)_{2}$-protohaem containing 50\% v/v ethanol. (Protohaem $=1.85 \times 10^{-5} \mathrm{M}$, $l=0.5 \mathrm{~cm}$.

$\left(\lambda=435 \mathrm{~m} \mu ., \epsilon \times 10^{-4}=11\right)$ is much stronger and lies nearer the red end of the spectrum than the cor- responding band $\left(\lambda=412 \mathrm{~m} \mu ., \epsilon \times 10^{-4}=7 \cdot 2\right)$ of ethanolic protohaem which, for the purposes of this paper, may be defined as a solution of haematin in a $50 \%(\mathrm{v} / \mathrm{v})$ mixture of $0 \cdot 1 \mathrm{~N} \cdot \mathrm{NaOH}$ and ethanol, reduced with $\mathrm{Na}_{2} \mathrm{~S}_{2} \mathrm{O}_{4}$ (Fig. 2). Dihydroxyl-protohaem can also be obtained if the ethanol is replaced by acetone or glycol monomethyl ether. The effect of ethanol is therefore not specific, and the formation of this compound requires the presence of almost any water-miscible solvent in addition to a high concentration of $\mathrm{NaOH}$.

The optimum conditions for the formation of dihydroxyl-protohaem were determined by varying the concentration of $(a)$ ethanol or $(b) \mathrm{NaOH}$, the concentrations of the other reagents remaining constant in both cases.

(a) Experiments with varying concentrations of ethanol. For this purpose a series of tubes was set up each containing $1 \mathrm{ml}$. of a $4.6 \times 10^{-4} \mathrm{M}$ solution of protohaematin in $1 \%(\mathrm{w} / \mathrm{v}) \mathrm{Na}_{2} \mathrm{CO}_{3}$ and $1 \mathrm{ml}$. of

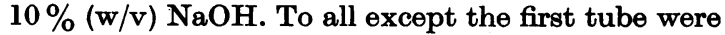
added varying quantities of absolute ethanol. The solutions were made up to a constant volume of $4 \mathrm{ml}$. with distilled water, reduced with dry $\mathrm{Na}_{2} \mathrm{~S}_{2} \mathrm{O}_{4}$ and examined spectroscopically.

The solutions containing $28.5 \%(v / v)$ or less of ethanol were opalescent, of a greenish brown colour and showed the spectrum of ordinary protohaem. When the concentration of ethanol reached $34 \%$ $(\mathrm{v} / \mathrm{v})$ the absorption spectrum of dihydroxyl-protohaem was faintly seen, superimposed on that of haem. With increasing concentrations of ethanol, the absorption bands of dihydroxyl-protohaem gradually replaced those of protohaem. The bands reached maximal intensity and the solution became clear and of a brownish pink colour only when it contained about $45 \%(v / v)$ ethanol. As a slightly higher concentration of ethanol did not affect the absorption spectrum of the compound, it was decided to add ethanol to $50 \%$ of the total volume of the solution in all subsequent experiments with dihydroxyl-protohaem.

(b) Experiments with varying concentrations of sodium hydroxide. In an attempt to determine whether the formation of the dihydroxyl-protohaem was a function of the $\mathrm{pH}$ of the solution, $\mathrm{Na}_{2} \mathrm{CO}_{3}$ was used as the alkali, but even saturation of a solution of ethanolic protohaem with $\mathrm{Na}_{2} \mathrm{CO}_{3}$ failed to produce the characteristic spectrum. It was found that only strong alkali such as $\mathrm{NaOH}$ would give rise to this spectrum and the minimal concentration for its complete formation was determined spectrophotometrically as follows. A stock solution of protohaematin was prepared containing $12.35 \mathrm{mg}$. protohaemin in $25 \mathrm{ml} .1 \%$ (w/v) $\mathrm{Na}_{2} \mathrm{CO}_{3}$ and a series of 10 tubes was set up each containing $1 \mathrm{ml}$. of the stock haematin solution and $3 \mathrm{ml}$. absolute ethanol. To tubes 2-10 were added varying amounts of 
$\mathrm{NaOH}$ ranging from $0.5 \mathrm{ml}$. $\mathrm{N}-\mathrm{NaOH}$ to $2 \mathrm{ml}$. $12 \mathrm{~N}-\mathrm{NaOH}$ and the volumes of all solutions were adjusted to $6 \mathrm{ml}$. with distilled water. The solutions were reduced with solid $\mathrm{Na}_{2} \mathrm{~S}_{2} \mathrm{O}_{4}$ and allowed to stand for about $30 \mathrm{~min}$. to allow full reduction to take place, especially in the more alkaline solutions. The maximum intensities of the absorption bands were measured for each solution, and it was found that a concentration of $1.33 \mathrm{~N}-\mathrm{NaOH}$ provided the optimum conditions for the formation of dihydroxylprotohaem.

The choice of solvent used for the formation of dihydroxyl-protohaem. In the reaction described above ethanol was used as originally specified by Dhéré \& Vegezzi (1916). The reaction, as has already been mentioned, is also given with acetone or glycol monomethyl ether, both of which are water-miscible solvents. But dioxan used in conjunction with $\mathrm{NaOH}$, though freely miscible with water, failed to givedihydroxyl-protohaem. When dioxan was added to a solution of haematin either in $1 \%(w / v) \mathrm{Na}_{2} \mathrm{CO}_{3}$ or in $0.1 \mathrm{~N}-\mathrm{NaOH}$ in the presence of $\mathrm{Na}_{2} \mathrm{~S}_{2} \mathrm{O}_{4}$, the solution becamea clear cherry red and its two-banded absorption spectrum closely resembled that of caffeine-haem (Keilin, 1943). However, dioxan greatly sharpened the absorption spectrum of COprotohaem both in aqueous and ethanolic solution, shifting the absorption bands a little towards the blue end of the spectrum, in contrast to the action of caffeine on CO-protohaem.

\section{Reactions of haems other than protohaem with sodium hydroxide}

Whereas protohaem requires ethanol or a similar solvent for the formation of dihydroxyl-protohaem, other haems such as deutero-, haemato- and urohaem form their corresponding dihydroxyl compounds without the addition of any organic solvent (Fig. 3). None of these haems, however, would give dihydroxyl compounds with $\mathrm{Na}_{2} \mathrm{CO}_{3}$ instead of $\mathrm{NaOH}$. The formation of dihydroxyl-haematohaem and dihydroxyl-urohaem with increasing concentrations of $\mathrm{NaOH}$ were followed spectrophotometrically as described for dihydroxyl-protohaem. The minimum concentration of $\mathrm{NaOH}$ required for the formation of dihydroxyl-haem compounds was found to be $1.33 \mathrm{~N}$ for protohaem in the presence of $50 \%$ ethanol and only $1.0 \mathrm{~N}$ for haemato- and urohaem in the complete absence of ethanol. The presence of ethanol, nevertheless, slightly sharpens and shifts the absorption bands of dihydroxylhaematohaem towards the red end of the spectrum. It affects similarly, but to a lesser degree, those of dihydroxyl-urohaem.

\section{Stoicheiometric relationships}

In order to determine the number of hydroxyl groups combined with each molecule of haem to give the hydroxyl compound, the haem was titrated with $\mathrm{NaOH}$ using the double wedge-trough method described by Keilin \& Hartree (1946). Haematohaem was selected for this experiment, since it does not require ethanol for the formation of its hydroxyl compound and is more stable to reoxidation by air than urohaem.

$3 \mathrm{ml}$. of a $6 \cdot 12 \times 10^{-4} \mathrm{M}$-solution of haematohaematin in $1 \% \mathrm{Na}_{2} \mathrm{CO}_{3}$ were diluted with $1 \%$ $\mathrm{Na}_{2} \mathrm{CO}_{3}$ to $25 \mathrm{ml}$. for one compartment of the double wedge trough, and with $1.5 \mathrm{M}-\mathrm{NaOH}$ for the other compartment, so that the haematin concentration was $7.35 \times 10^{-5} \mathrm{M}$ in each compartment of the

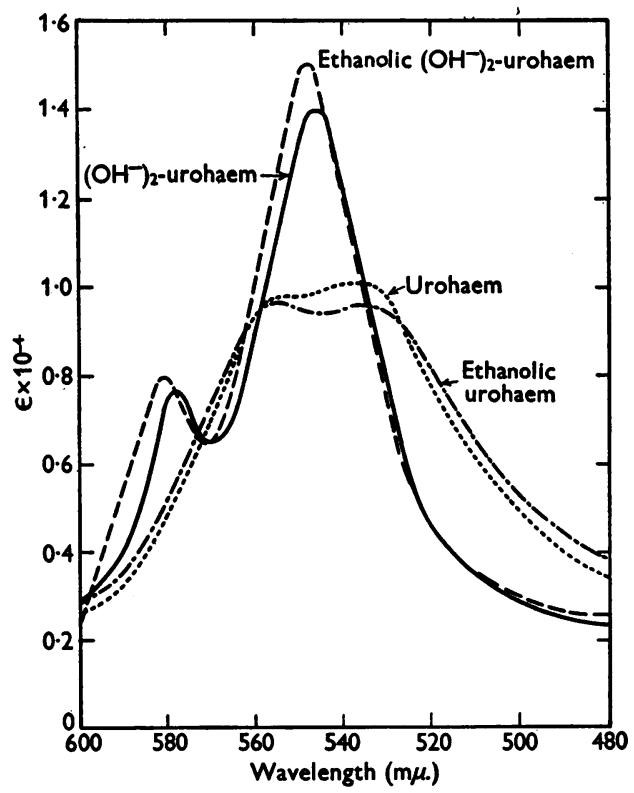

Fig. 3. Absorption bands in the visible region of the spectrum of $\left(\mathrm{OH}^{-}\right)_{2}$-urohaem, urohaem, ethanolic urohaem and ethanolic $\left(\mathrm{OH}^{-}\right)_{2}$-urohaem. The two latter solutions contain $50 \% \mathrm{v} / \mathrm{v}$ ethanol. (Urohaem $=1.263 \times 10^{-4} \mathrm{M}$, $l=0.5 \mathrm{~cm}$.)

trough. The volume $(v)$ of $6 \cdot 12 \times 10^{-4} \mathrm{M}$-haematohaematin for the required optical depth in the cup was calculated as follows: $v=\pi r^{2}\left(c_{1} l / c_{2}\right)$, where $c_{1}$ and $c_{2}=$ haematohaematin concentrations in trough and cup, respectively, $l=$ light path in trough $(2 \cdot 1 \mathrm{~cm}$.) and $r=$ radius of cup. All the solutions were reduced with $\mathrm{Na}_{2} \mathrm{~S}_{2} \mathrm{O}_{4}$ and the haematohaem in the cup was matched against the broad end of the haematohaem compartment of the trough which was at zero position, the light intensities of the two spectra having been carefully equalized. The haem in the cup was then titrated with $\mathrm{N}-\mathrm{NaOH}$ and the spectrum of the solution was matched, after each addition of $\mathrm{NaOH}$, against the optical mixture of haematohaem and its hydroxyl compound in the two compartments of the trough. The concentrations 
Table 1. The relation between the sodium hydroxide concentration and percentage formation of dihydroxylhaematohaem as determined experimentally and as calculated from the results by the method of least squares

\begin{tabular}{|c|c|c|}
\hline \multirow{2}{*}{$\begin{array}{c}\text { Total } \mathrm{NaOH} \\
\text { added } \\
\text { (ml.) }\end{array}$} & \multicolumn{2}{|c|}{$\mathrm{NaOH}$ concentration in solution } \\
\hline & $\mathbf{M}$ & $\log \mathbf{M}$ \\
\hline 0.05 & $0 \cdot 238$ & -0.6231 \\
\hline $0 \cdot 10$ & $0 \cdot 384$ & -0.4157 \\
\hline $0 \cdot 25$ & 0.612 & $-0 \cdot 2132$ \\
\hline $0 \cdot 35$ & $0 \cdot 688$ & $-0 \cdot 1624$ \\
\hline $0 \cdot 45$ & $0 \cdot 740$ & $-0 \cdot 1308$ \\
\hline 0.55 & $0 \cdot 775$ & -0.1107 \\
\hline $0 \cdot 70$ & $0 \cdot 814$ & -0.0894 \\
\hline $0 \cdot 80$ & 0.825 & -0.0835 \\
\hline
\end{tabular}

of haematohaematin in the cup and trough were specially selected so that the spectrum of the haematohaem was clearly visible, while that of the fully formed hydroxyl compound in the trough

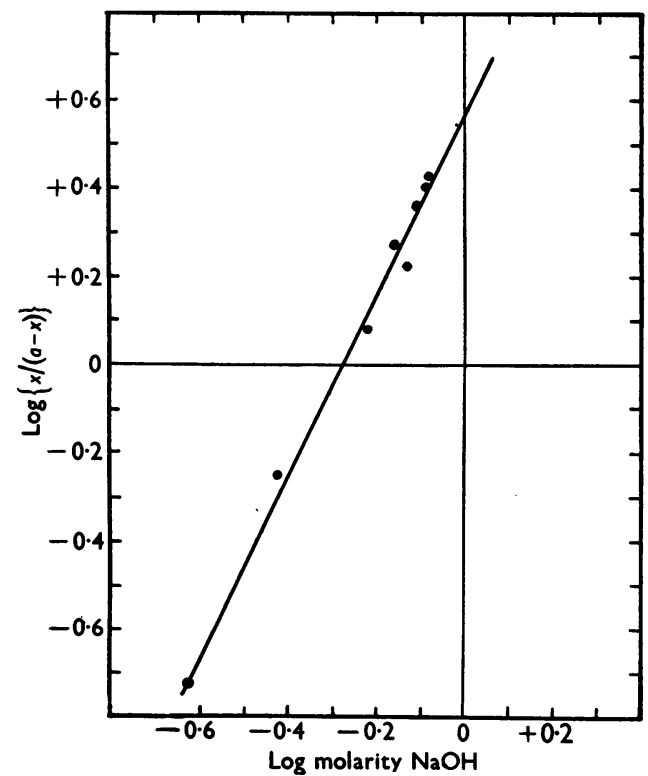

Fig. 4. Relationship between log molarity of $\mathrm{NaOH}$ and $\log \{x /(a-x)\}$, where $x=$ percentage formation of $\left(\mathrm{OH}^{-}\right)_{2^{-}}$ haematohaem and $a-x=$ percentage of free haematohaem. Experimental results are represented by dots. Results calculated from the equation

$$
\log \{x /(a-x)\}=n \log \left(\mathrm{OH}^{-}\right)-\log K,
$$

where $n=2 \cdot 08$ and $K=0 \cdot 264$, are represented by the straight line.

(containing excess $\mathrm{NaOH}$ ) was not too intense to prevent matching with the solution in the cup. It was thus possible to determine the percentage formation of the hydroxyl compound at different concentrations of $\mathrm{NaOH}$ and the results are given in Table 1.

If the reaction is represented by the following equation:

$$
\mathrm{Haem}+n\left(\mathrm{OH}^{-}\right) \rightleftharpoons \mathrm{Haem}\left(\mathrm{OH}^{-}\right)_{n},
$$

Percentage

ormation of Percentage compound free haem

$\begin{array}{cc}(x) & (a-x) \\ 16 & 84 \\ 36 & 64 \\ 56 & 44 \\ 65 & 35 \\ 62 & 38 \\ 70 & 30 \\ 72 & 28 \\ 73 & 27\end{array}$

$\begin{array}{cc}\overbrace{\text { Determined }}^{\text {experimentally }} & \text { Calculated } \\ -0.7201 & -0.718 \\ -0.2495 & -0.286 \\ +0.0864 & +0.135 \\ +0.2669 & +0.240 \\ +0.2253 & +0.306 \\ +0.3674 & +0.348 \\ +0.4099 & +0.392 \\ +0.4314 & +0.409\end{array}$

then from the law of mass action

$$
\frac{\left[\mathrm{Haem}\left(\mathrm{OH}^{-}\right)_{n}\right]}{[\mathrm{Hrem}]\left[\mathrm{OH}^{-}\right]^{n}}=K \text {. }
$$

If $x$ represents the amount of the hydroxyl compound formed and $a$ represents the total haem present, then

$$
\frac{x}{(a-x)\left[\mathrm{OH}^{-}\right]^{n}}=K,
$$

whence $\log \{x /(a-x)\}=n \log \left[\mathrm{OH}^{-}\right]-\log K$.

By applying the method of least squares to the values obtained for $\log \{x /(a-x)\}$ and $\log \left[\mathrm{OH}^{-}\right]$, it was found that $n=2.08$ and $\log \left[\mathrm{OH}^{-}\right]=-0.278$ at $50 \%$ formation of the hydroxyl compound while $k=0 \cdot 264$, since under these conditions $\log K=n \log \left[\mathrm{OH}^{-}\right]$. (This value for $k$ does not take into account the very slight depression of $\left[\mathrm{OH}^{-}\right]$due to the presence of $\mathrm{Na}_{2} \mathrm{~S}_{2} \mathrm{O}_{4}$, nor the activity coefficient of $\mathrm{NaOH}$.) On substituting these values for $n$ and $K$ in the above equation, the theoretical values of $\log \{x /(a-x)\}$ for different concentrations of $\mathrm{NaOH}$ can be calculated. These calculated values, together with those obtained experimentally, are shown in Fig. 4. This experiment shows conclusively that in the hydroxyl-haematohaem compound the haem $\mathrm{Fe}$ combines with two hydroxyl groups, and since the corresponding compounds obtained with other haems have similar properties, all these compounds may be described under the name of dihydroxyl-haems.

\section{Reactions of dihydroxyl-haems with carbon monoxide}

When a current of $\mathrm{CO}$ is passed through a solution of dihydroxyl-protohaem, the latter assumes a bright cherry-red colour and its absorption bands are shifted to the following positions : $\alpha, 563 ; \beta, 532$ and $\gamma, 410 \mathrm{~m} \mu$. This spectrum corresponds to that of a solution of ethanolic CO-protohaem (Fig. 5). Similarly, the CO derivatives of dihydroxyl-haematohaem and dihydroxyl-urohaem in aqueous solution are indistinguishable from their respective aqueous solutions of CO-haems.

At this point it may be interesting to recall Dhéré \& Vegezzi's (1916) experiments on the 
reduction of acid and alkaline ethanolic solutions of protohaematin in open and sealed tubes. They invariably found that in sealed tubes the absorption spectra of the solutions were very different from those in open tubes and, although the latter contained an excess of reducer, they believed at first that complete reduction could only be obtained in sealed tubes. However, later Dhéré (1927) recognized that the difference was not due to the more complete reduction of the solutions in sealed tubes but to the formation within them of CO-protohaem, the $\mathrm{CO}$ having been produced by incomplete combustion of ethanol during the process of sealing the tubes.

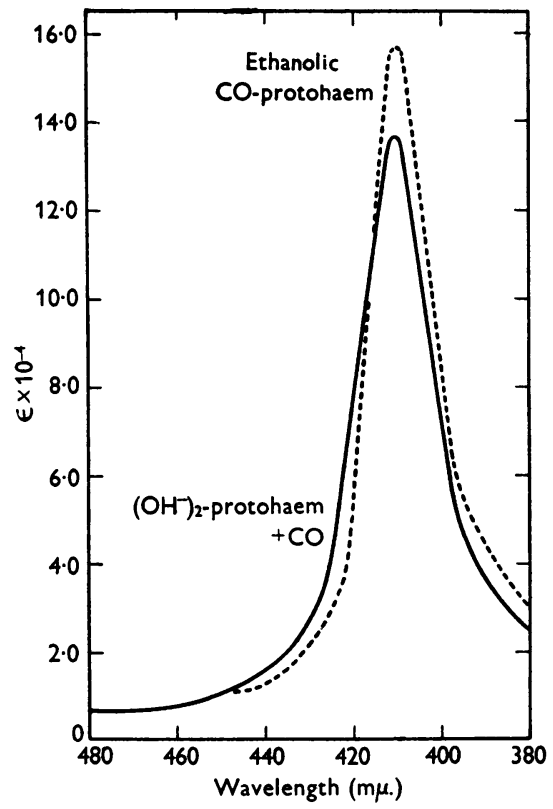

Fig. 5. Absorption spectra showing $\gamma$-bands of $\left(\mathrm{OH}^{-}\right)_{2}$ protohaem + CO and of CO-protohaem containing $50 \%$ $\mathrm{v} / \mathrm{v}$ ethanol. (Protohaem $=1.85 \times 10^{-5} \mathrm{M}, l=0.5 \mathrm{~cm}$.)

\section{The magnetic susceptibility of dihydroxyl-urohaem}

The method of Gouy (1889) was adapted for use with an 'Alcomax' permanent magnet giving a uniform field of 10,600 gauss within a gap of $6 \mathrm{~mm}$. The magnet was mounted on a jack so that it could be moved vertically with precision and without any lateral displacement of the magnet. The magnet and jack were enclosed in a glass case and kept at constant temperature $\left(20 \cdot 5^{\circ}\right)$. A narrow glass tube $(10 \mathrm{~cm}$. long, $2 \mathrm{~mm}$. internal diam. and $0.3 \mathrm{ml}$. capacity), fitted with glass suspending hooks at the open end and closed with a rubber cap, was hung on a platinum wire from the pan of the balance and weighed with its lower end between the pole pieces of the magnet. The magnet was then lowered about $25 \mathrm{~cm}$. by means of the jack, and the tube, now in a negligible magnetic field, was weighed again. The increase in weight, $\Delta w$, was then similarly determined $(a)$ with the tube full of distilled water, (b) with the tube filled with the haem solution under investigation, and (c) with the tube filled with the solvent (i.e. all the constituents except the haemin). These changes in weight were each corrected for the change in weight of the empty tube $\Delta w$, and are represented as $\Delta w_{\mathrm{H}_{2} \mathrm{O}}, \Delta w_{\text {soln. }}$ and $\Delta w_{\text {solv. }}$ respectively.

The mass susceptibility $\chi$ of the solution was calculated according to the following formula, derived from the equation given by Trew \& Watkins (1933):

$$
10^{6} \chi_{\text {soln. }}=\left(10^{6} \chi_{\mathrm{H}_{2} \mathrm{O}}-\frac{K_{\mathrm{alr}}}{d_{\mathrm{H}_{2} \mathrm{O}}}\right) \frac{\Delta w_{\text {Boln. }} \cdot d_{\mathrm{H}_{2} \mathrm{O}}}{\Delta w_{\mathrm{B}_{2} \mathrm{O}} \cdot d_{\text {soln. }}}+\frac{K_{\mathrm{atr}}}{d_{\text {soln. }}},
$$

where $\chi_{\mathrm{H}_{2} \mathrm{O}}$, the mass susceptibility of water $=-0.72 \times 10^{-6}$; $K_{\text {air }}$, the volume susceptibility of air $=+0.0294 \times 10^{-6}$; and $d=$ specific gravity.

The value of $10^{6} \chi_{\text {sol. }}$. was similarly calculated and the mass susceptibility of the solute (haem) was obtained from:

$$
\chi_{\text {soln. }}=c \chi_{\text {solute }}+(1-c) \chi_{\text {solv. }}
$$

where $c=$ wt. of dissolved haemin/wt. of solution.

The magnetic moment $\mu_{B}$ (in Bohr magnetons) is then given by

$$
\mu_{\mathrm{B}}=2 \cdot 84 \sqrt{ }(\chi M T) \text {, }
$$

where $\chi=$ mass susceptibility of haem compound, $M=$ mol.wt. of haemin, $T=$ absolute temperature.

Urohaem was selected for these experiments instead of protohaem in view of its greater solubility and the ease in bringing the reaction to completion. The magnetic susceptibilities of protohaematin, dicyan-protohaem and dicyanurohaem were also determined by the same method. This was done in order: (1) to test the accuracy of the procedure, (2) to test the suitability of the urohaem preparation for such measurements, and (3) to provide the data necessary for comparison with those obtained for dihydroxyl-urohaem.

The solutions were made up as follows:

Protohaematin. Protohaemin (50 mg.) was dissolved in $5 \mathrm{ml} .0 \cdot 2 \mathrm{~N}-\mathrm{NaOH}$ containing about $30 \%$ sucrose (Pauling \& Coryell, 1936).

Dicyan-protohaem. Protohaemin (60 mg.) was dissolved in about $6 \mathrm{ml}$. of the above sucrose- $\mathrm{NaOH}$ solution. $\mathrm{KCN}$ (300 mg.) and $\mathrm{Na}_{2} \mathrm{~S}_{2} \mathrm{O}_{4}$ (100 mg.) were then added and the volume of the solution was made up to $10 \mathrm{ml}$. with sucrose$\mathrm{NaOH}$. (This order was adopted to avoid the gelatinous precipitate which tended to form if the solid haemin was added last.)

Dicyan-urohaem. Urohaemin (20 mg.), KCN (30 mg.) and $\mathrm{Na}_{2} \mathrm{~S}_{2} \mathrm{O}_{4}(20 \mathrm{mg}$.) were dissolved in that order in $1 \mathrm{ml} .1 \%$ $\mathrm{Na}_{2} \mathrm{CO}_{3}$ contained in a small, stoppered, graduated bottle with a narrow neck to minimize the air space above the fluid. The solution was transferred by means of a Pasteur pipette to the magnetic susceptibility tube which was completely filled and closed with the rubber cap. These precautions to exclude contact with air were necessary on account of the extreme ease with which the reduced urohaem compounds are oxidized in air.

Dihydroxyl-urohaem. Urohaemin (20.7 mg.) and $\mathrm{Na}_{2} \mathrm{~S}_{2} \mathrm{O}_{4}$ (20.3 mg.) were dissolved in $1 \mathrm{ml}$. $\mathrm{M}-\mathrm{NaOH}$, observing the precautions described above.

The results of these experiments, which were carried out at $20.5^{\circ}$ and which are summarized in Table 2, show that the Fe in dihydroxyl-urohaem has a much higher magnetic moment than the $\mathrm{Fe}$ in dicyan-urohaem. The values obtained for protohaematin and dicyan-protohaem are in agreement with those given by Pauling \& Coryell (1936) for 
Table 2. The values for $\Delta w$ and specific gravity $(d)$ of water, solvent and solution, and the molar susceptibility $\chi_{m}$ and magnetic moment $\mu_{B}$ (in Bohr magnetons) of protohaematin, dicyan-protohaem, dicyan-urohaem and dihydroxyl-urohaem

(Approx. 30\% sucrose was used only in the case of protohaematin and dicyan-protohaem.)

Proto-

haematin

Dicyan-

protohaem

Dicyan-

urohaem

Dihydroxylurohrem

$$
10
$$

-

30

$20 \quad 50$

$20 \cdot 1$

50

$\mathrm{N}-\mathrm{NaOH}$
Compound $\begin{gathered}\mathrm{Na}_{2} \mathrm{~S}_{2} \mathrm{O}_{4} \\ (\mathrm{mg} / \mathrm{ml} .)\end{gathered} \underset{\begin{array}{c}\mathrm{KCN} \\ (\mathrm{mg} / \mathrm{ml} .)\end{array}}{\begin{array}{c}\text { Alkali and } \\ \text { sucrose }\end{array}}$

\section{Solvent}

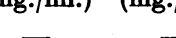

$30 \%$ sucrose,

0.2 $\mathrm{N}-\mathrm{NaOH}$

$30 \%$ sucrose,
0.2 N-NaOH

$1 \% \mathrm{Na}_{2} \mathrm{CO}_{3}$

$2 \cdot 44$

both compounds and by Haurowitz \& Kittel (1933) for protohaematin. The relatively high values obtained for both the urohaem compounds are probably due to some degree of oxidation that occurred in spite of the precautions taken to avoid it.

\section{DISCUSSION}

The solubility of a haemin derivative depends very largely on that of its parent porphyrin and the porphyrins differ greatly in the nature of their side chains. Carboxyl groups in general confer solubility on the molecule so that uroporphyrin, with its eight carboxylgroups, is the most soluble of the porphyrins. Haematoporphyrin, with $-\mathrm{CH}(\mathrm{OH}) . \mathrm{CH}_{3}$ groups in positions 2 and 4 , is more soluble than protoporphyrin which has vinyl groups $\left(-\mathrm{CH}=\mathrm{CH}_{2}\right)$ in these positions. Haemin derivatives also differ in solubility according to the valency of their iron. Thus the ferrous compounds such as haem and haemochromogen are less soluble than their ferric forms, haematin and parahaematin. It is also interesting to note that CO-haem is more soluble than free haem.

It is well known that the solution or dispersion of molecular aggregates of a pigment is accompanied by an intensification of its absorption bands and by a more or less marked shift of these bands towards the blue end of the spectrum. In fact, the addition of ethanol intensifies the absorption bands of haems, and this intensification, which varies inversely with the solubility of the haem in dilute aqueous alkali, is therefore more marked for proto- than for haematohaem and is very feeble in the case of urohaem.

The conditions required for the formation of dihydroxyl-haem compounds are $(a)$ a high concentration of alkali (1-1.33 $\mathrm{N}-\mathrm{NaOH})$, and $(b)$ a certain degree of solubility of the haem. In the case of protohaem, which is relatively insoluble, the required degree of solubility can only be obtained by the addition of an appreciable amount of ethanol (up to $50 \%$ ) or of another water-miscible solvent such as acetone or glycol monomethyl ether. On the other hand, ethanol is not required for the formation of dihydroxyl-urohaem and, when added, hardly

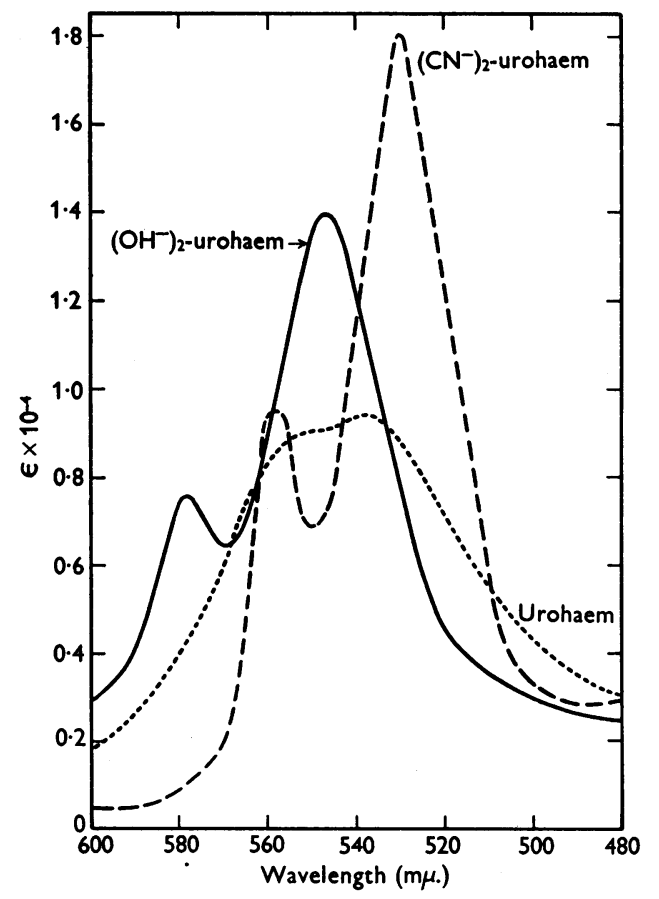

Fig. 6. Absorption bands in the visible region of the spectrum of $\left(\mathrm{OH}^{-}\right)_{2}$-urohaem, urohaem (urohaem $=1.263 \times 10^{-4} \mathrm{M}$, $l=0.5 \mathrm{~cm}$.) and $\left(\mathrm{CN}^{-}\right)_{2}$-urohaem (urohaem $=6.61 \times 10^{-5} \mathrm{M}$, $l=0.25 \mathrm{~cm}$.).

affects the spectrum, as urohaem itself is very soluble in very dilute alkali. In this respect haematohaem occupies an intermediate position between proto- and uro-haem. Although dihydroxyl-haematohaem, with its characteristic absorption spectrum, may be formed in the complete absence of ethanol, the addition of the latter intensifies its bands and 
shifts them slightly towards the red end of the spectrum. This change is brought about by a simultaneous increase in the solubility of the haematohaem in presence of ethanol and a decrease in that of its dihydroxyl compound.

Information as to the nature of the hydroxyl-haem compound was obtained in two ways: (1) Directly, by titrating the haem with $\mathrm{NaOH}$ and determining its dissociation constant. This showed that two hydroxyl groups were combined with each atom of haem $\mathrm{Fe}$ (see Table 1 and Fig. 4). (2) Indirectly, by comparing its properties with those of other haem derivatives such as haem, caffeine-haem, haemochromogens, monocyan- and dicyan-haem and monocarbylamine- and dicarbylamine-haem. The absorption spectra of dihydroxyl-haems and the corresponding dicyan-haems bear a striking resemblance to each other (Figs. 1 and 6). This resemblance, which can be seen in the visible and the violet regions of the spectrum, applies to the general pattern of the absorption spectrum as reflected in the relative intensities of the bands. The fact that the general spectral pattern common to dihydroxyl-haems, dicyan-haems and dicarbylamine-haems differs so markedly from those of all other haematin derivatives suggests that these compounds have similar structures.

Spectroscopic and potentiometric investigations (see Anson \& Mirsky, 1925; Hill, 1929; Davies, 1940; Shack \& Clark, 1947) suggest that the structures of different haem (ferrous) compounds can be represented as follows (in these schematic representations the lines joining the haem Fe to the reacting groups do not indicate the nature of the valency bonds; the four valencies directed towards the pyrrole nitrogen atoms are omitted):

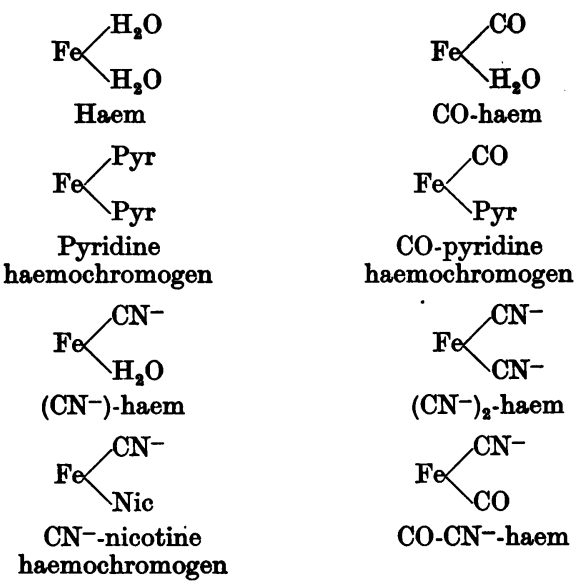

By analogy with dicyan-haem, the structure of dihydroxyl-haem may therefore be represented as

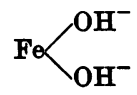

The study of Dhéré \& Vegezzi's (1916) compound, apart from its structural relationship with different haem derivatives, is important in two other respects. (1) Optical and magnetic studies on different haematin compounds have suggested the existence of a certain empirical relationship between the pattern of their absorption spectra and the type of iron bonding in each compound as determined by magnetic susceptibility measurements (Theorell, 1942 ; Hartree, 1947). In this respect the dihydroxylurohaem presents a striking exception. Although the absorption spectra of dihydroxyl-urohaem and dicyan-urohaem are of the same pattern, the magnetic measurements show fundamental structural differences. While the $\mathrm{Fe}$ atom in dihydroxylurohaem is linked by ionic bonds, with the result that the compound shows the high magnetic moment characteristic of free haems, the bonds in dicyanurohaem are essentially covalent and the low magnetic moment brings it into line with the diamagnetic haemochromogens. In view of the strict analogies existing between the corresponding compounds of different haems it is reasonable to assume that the results obtained for urohaem may be taken to apply to dihydroxyl compounds of other haems. (2) Strong alkalis, with or without ethanol, are often used in studying the reactions between haems and different nitrogenous substances. However, unless one is familiar with the absorption spectrum of dihydroxyl-haem and aware of its possible appearance during the course of the reaction, the main absorption band of this compound can easily be mistaken for that of a partly developed haemochromogen, since its strong $\beta$-band can be seen where the $\alpha$-band of the haemochromogen is expected to appear. This aspect of the problem will be discussed more fully elsewhere.

\section{SUMMARY}

1. It is shown that Dhéré \& Vegezzi's (1916) 'alkaline haemochromogen' is a compound in which the haem iron is combined with two hydroxyl groups and it is therefore named dihydroxyl-haem.

2. For the full formation of dihydroxyl-protohaem $1 \cdot 33 \mathrm{~N}-\mathrm{NaOH}$ and $50 \%(\mathrm{v} / \mathrm{v})$ ethanol are required. Ethanol, however, can bereplaced by other water-miscible organic solvents such as acetone and glycol monomethyl ether, but not by dioxan.

3. In the case of haematohaem and urohaem the corresponding dihydroxyl compounds can be obtained in complete absence of an organic solvent, although the absorption spectrum of dihydroxylhaematohaem is reinforced by ethanol.

4. The dissociation constant $K$ for dihydroxylhaematohaem is 0.264 at $20^{\circ}$.

5. The absorption spectrum of dihydroxyl-haem differs markedly from those of haem, caffeine-haem, 
haemochromogens and monocyan-haem, but shows a striking resemblance to those of dicyan-haem and dicarbylamine-haem.

6. The bearing of the similarity of the absorption spectra of dicarbylamine-haem, dicyan-haem and dihydroxyl-haem on the structure of this compound is discussed.

7. Dihydroxyl-haem, unlike dicyan-haem, is paramagnetic, and when treated with carbon monoxide gives CO-haem.
8. The knowledge of the absorption spectrum and other properties of the dihydroxyl-haem compound is of special importance for the study of the reactions between haems and nitrogenous compounds.

I wish to thank Dr R. Hill, F.R.S., for his valuable suggestions and interest in this work; and Dr E. F. Hartree, for advice regarding the magnetic susceptibility determinations. Thanks are also due to the Medical Research Council for a personal grant.

\title{
REFERENCES
}

Anson, M. L. \& Mirsky, A. E. (1925). J. Physiol. 60, 50.

Davies, T. H. (1940). J. biol. Chem. 135, 597.

Dhéré, Ch. (1927). C.R. Soc. Biol., Paris, 97, 1660.

Dhéré, Ch. \& Vegezzi, G. (1916). C.R. Acad. Sci., Paris, 163, 209.

Fischer, H. \& Orth, H. (1934). Die Chemie des Pyrrols, 2. Leipzig: Akademische Verlagsgesellschaft.

Gouy, G. (1889). C.R. Acad. Sci., Paris, 109, 935.

Hartree, E. F. (1947). Rep. Progr. Chem. 43, 287.

Haurowitz, F. \& Kittel, H. (1933). Ber. dtsch. chem. Ges. 66, 1046.

Hill, R. (1926). Proc. Roy. Soc. B, 100, 419.

Hill, R. (1929). Proc. Roy. Soc. B, 105, 112.

Keilin, J. (1943). Biochem. J. 37, 281.
Keilin, J. (1949). Biochem. J, 45, 440.

Keilin, D. \& Hartreø, E. F. (1946). Nature, Lond., 157, 210:

Nencki, M. \& Seiber, N. (1888). Arch. exp. Path. Pharmak. 24, 430.

Pauling, L. \& Coryell, C. D. (1936). Proc. nat. Acad. Sci., Wash., 22, 159.

Schalfejeff, M. (1885). Abstract in Ber. dtsch. chem. Ges. 18 (Referat Bd.), 232.

Shack, J. \& Clark, W. Mansfield (1947). J. biol. Chem. 171, 143.

Theorell, H. (1942). Arkiv. Kemi Min. Geol. 16 A, no. 3.

Trew, V. C. G. \& Watkins, G. M. C. (1933). Trans. Faraday Soc. 29, 1310.

\section{The Semi-micro Estimation of Lactose Alone and in the Presence of Other Sugars}

\author{
By F. H. MALPRESS AND A. B. MORRISON \\ Department of Biochemistry, Queen's University, Belfast
}

(Received 13 April 1949)

In the course of studies on the intermediary metabolism of the mammary gland, and in particular the investigation of lactose formation in incubated tissues, it became necessary to devise improved methods for estimating this sugar in the presence of glucose and glycogen. The methods hitherto available for the estimation of small amounts of lactose, alone or in the presence of other sugars, are laborious and unsatisfactory. Those normally used for estimating lactose in milk suffer from a lack of specificity which makes them valueless in any investigation where mixtures of sugars and related carbohydrates are likely to be found, while those designed for more general use rely on the complementary techniques of yeast fermentation, for removing fermentable sugars, and the use either of reduction methods or of a lactose-fermenting yeast to give the lactose equivalent of the residual fermented solutions (Grant,
1935; Scott \& West, 1936). The application of paper chromatography to the separation and characterization of sugars in mixtures (Partridge, 1946, 1948), and the adaptation of this method to the estimation of sugars (Flood, Hurst \& Jones, 1947; Hawthorne, 1947), are advances of great value, but the techniques are time-consuming and, for other reasons, frequently unsuited for routine work (see Westall, 1948).

In the hope of obtaining a method which would be more convenient in practice and yet have greater specificity than those ultimately dependent on estimations of reducing power, the colour reaction given by lactose with methylamine in alkaline solution, first observed by Fearon (1942) and later used in qualitative studies for detecting lactosuria in pregnancy and the early puerperium (Harwood, 1946; Archer \& Haram, 1948), has been developed into a quantitative method. Fearon found that the 